\title{
Transatlantica
}

Revue d'études américaines. American Studies Journal

1 | 2017

Morphing Bodies: Strategies of Embodiment in

Contemporary US Cultural Practices

\section{Edward Hopper va au cinéma}

Jean-Loup Bourget

\section{(2) OpenEdition}

Journals

Édition électronique

URL : https://journals.openedition.org/transatlantica/8441

DOI : 10.4000/transatlantica.8441

ISSN : 1765-2766

Éditeur

Association française d'Etudes Américaines (AFEA)

Référence électronique

Jean-Loup Bourget, «Edward Hopper va au cinéma », Transatlantica [En ligne], 1 | 2017, mis en ligne le 27 novembre 2018, consulté le 20 mai 2021. URL : http://journals.openedition.org/transatlantica/8441 ; DOI : https://doi.org/10.4000/transatlantica.8441

Ce document a été généré automatiquement le 20 mai 2021.

\section{(c) $(1) \odot$}

Transatlantica - Revue d'études américaines est mise à disposition selon les termes de la licence Creative Commons Attribution - Pas d'Utilisation Commerciale - Pas de Modification 4.0 International. 


\section{Edward Hopper va au cinéma}

\section{Jean-Loup Bourget}

\section{Edward Hopper (1882-1967), New York Movie, 1939}

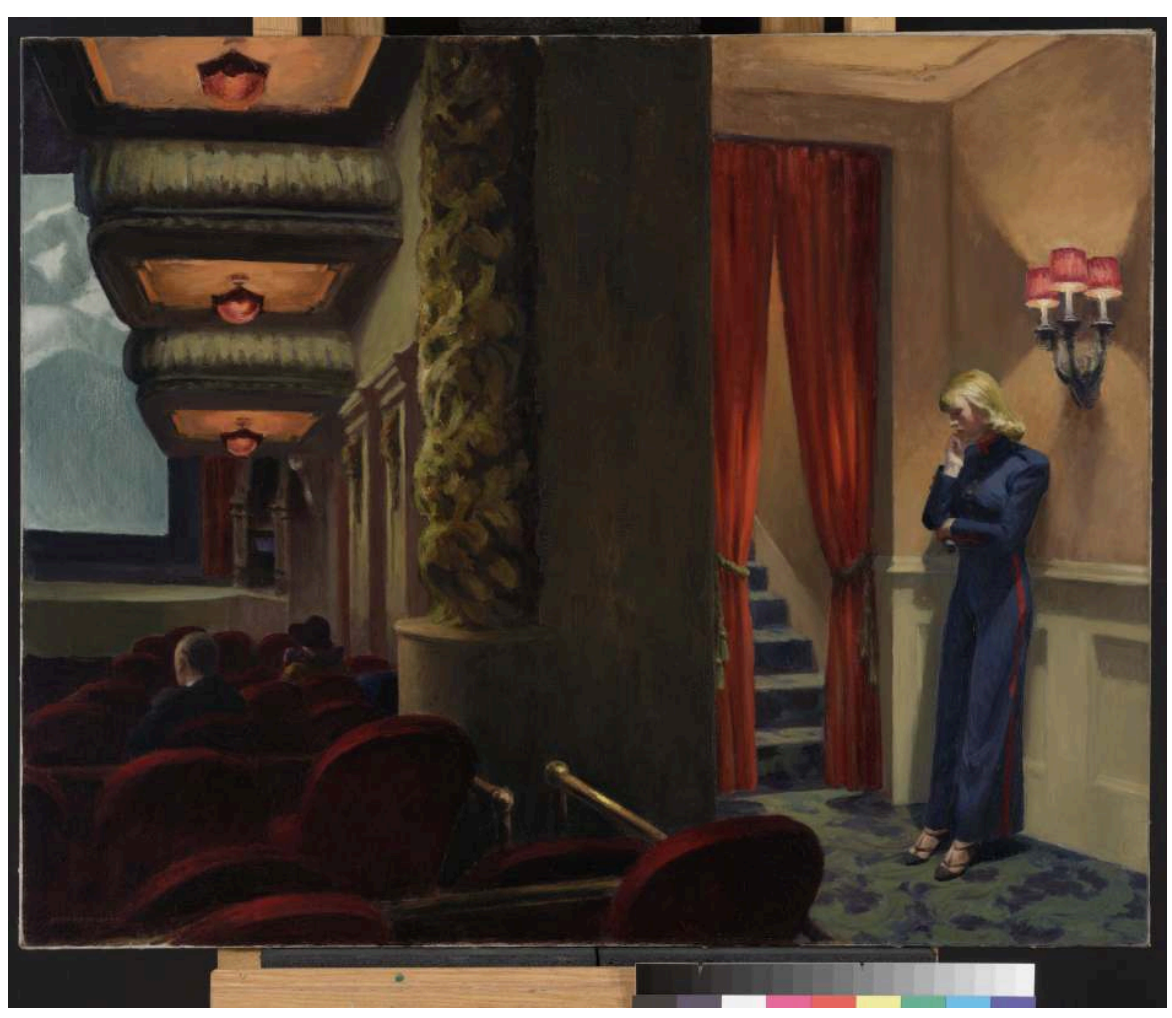

New York, Museum of Modern Art (MoMA). Huile sur toile, $81.9 x$

101.9 cm. Don anonyme. Acc. n.: 396.1941

(c) 2018. Digital

image, The Museum of Modern Art, New York/Scala, Florence 
1 New York Movie (MoMA, 1939) est dans l'œuvre d'Edward Hopper un de mes personal favorites, d'abord pour l'évidente raison qu'il satisfait à la fois mon goût de la peinture et mon intérêt pour le cinéma. Sans atteindre à la notoriété de Nighthawks (1942), ce tableau est, au moins en Europe, en passe de devenir une "icône " de la peinture américaine $\mathrm{du} \mathrm{xx}^{\mathrm{e}}$ siècle, comme l'atteste par exemple sa reproduction en couverture de trois ouvrages récents, Théâtre et cinéma. Le croisement des imaginaires (sous la direction de Marguerite Chabrol et Tiphaine Karsenti, Presses Universitaires de Rennes, 2013), Une histoire culturelle des États-Unis de Jean Kempf (Armand Colin, 2015) et Bajo el signo de la melancolía. Cine, desencanto y aflicción de Santos Zunzunegui (Madrid, Cátedra, 2017). ${ }^{1}$ Cette vogue est relativement nouvelle : je me souviens d'une visite au MoMA, en 2012, où, relégué au fond d'un couloir en cul-de-sac, après le trop célèbre Christina's World (1948) d'Andrew Wyeth, New York Movie était presque introuvable. ${ }^{2}$

2 De la Solitary Figure in a Theater (Whitney, v. 1902-04) et de la gravure The Balcony (ou The Movies, 1928) jusqu'à Intermission (coll. privée, 1963) et Two Comedians (coll. privée, 1966), son dernier tableau, on sait que Hopper a beaucoup représenté des lieux de spectacle, qu'il s'agisse de théâtres (Two on the Aisle, Toledo, 1927 ; The Sheridan Theater, Newark, 1937), de cinémas ou de music-halls, entre lesquels il n'est pas toujours facile de distinguer. La gamme va du plus chic (les spectateurs en tenue de soirée de First Row Orchestra, Hirshhorn, 1951) au plus vulgaire (le burlesque de Girlie Show, coll. privée, 1941).

3 Ce qui frappe d'abord dans New York Movie est moins le fait que la salle de cinéma soit quasiment déserte (on n'aperçoit que deux spectateurs isolés) - c'est souvent le cas dans les tableaux de Hopper - que la nature singulière de l'espace intérieur représenté. Le sombre et massif mur-pilier central divise cet espace en deux sous-espaces ou "scènes ", celle de la salle de projection et celle de son bas-côté, ou de sa coulisse, qui paraissent juxtaposées et semblent appartenir à des espaces distincts. Cette impression est renforcée tant par le recours à la perspective que par l'emploi de la couleur. Tandis que dans la partie gauche, l'œil, guidé par la perspective des trois plafonniers, explore l'espace de la salle jusqu'à l'écran qui en constitue le fond, à droite l'esquisse de l'escalier invite à une exploration du même type, mais celle-ci est bloquée ou frustrée par les rideaux rouges et la mystérieuse lumière qui émane d'eux. Malgré les plafonniers et l'écran, la partie gauche est globalement sombre, au contraire de la partie droite, qui bénéficie, grâce à l'applique murale, d'un éclairage qui, se concentrant sur la chevelure blonde de l'ouvreuse, attire nécessairement l'œil du «spectateur». Ces quatre sources de lumière (applique, lumière provenant de l'escalier, plafonniers et écran) sont notées avec soin dans le livre de comptes (ledger folio) ou pour mieux dire le livre de vérité dans lequel Hopper, à la manière de claude Gellée le Lorrain (1600-1680), consignait la trace de ses tableaux, sous la forme d'un croquis accompagné par une description détaillée rédigée par le peintre et/ou son épouse Josephine ; l'énumération des sources de lumière s'y conclut par l'observation : «Canvas much darker than this sketch. Brightest point, girl's hair \& glint on brass rail » (ledger folio, vol. II, 27, reproduit dans Lyons, 1997, 50, et dans Foster, 2013, 122).

4 Confirmant de façon décisive que les deux «scènes " se tournent pour ainsi dire le dos et regardent dans des directions opposées, l'ouvreuse, de façon très évidente, ne regarde pas le film projeté sur l'écran, elle lui est absente et paraît entièrement absorbée dans une sorte de rêverie ou de méditation tout intérieure. Le tableau dans son ensemble suscite donc une impression de solitude, de silence, de mélancolie. Je n'irai pas jusqu'à 
parler d' «anxiété » comme le faisait le sous-titre de l'exposition de l'Orangerie (The Age of Anxiety) : ce serait là, me semble-t-il, procéder à une surinterprétation arbitraire. Hopper, on le sait, refuse l'anecdote, même si le secret de son succès posthume réside pour une large part dans la tendance du spectateur à projeter ses désirs et ses fantasmes sur la scène muette et peu explicite des tableaux. Ce qui est sûr est qu'ici Hopper dépeint une salle de cinéma bien éloignée des images collectives, dynamiques et criardes qui sont proposées à la même époque par son ami Reginald Marsh (Twenty Cent Movie, 1936, Whitney, aussi dans l'exposition) ou Thomas Hart Benton (America Today, 1930-32, Met Fifth Avenue ; Arts of Life in America, 1932, New Britain, Conn.), ou encore de celles plus féeriques de Florine Stettheimer (The Cathedrals of Broadway, 1929, Met Fifth).

Comme il est fréquent chez Hopper, ce qui contribue à la beauté du tableau (et à son caractère à mes yeux plus mélancolique qu'« anxieux » ou angoissé) est la volupté de la couleur, évoquée elle aussi avec précision dans le ledger folio : «Interior dark rich color, dark garnet, pink lights from under boxes, pink shades or wall lights on $R$ [ight]. wall, dark red plush chairs where color visible, garnet curtain at R[ight]. [...] " (Lyons, 1997, 50, Foster, 2013, 122). Le grenat dominant des sièges et des rideaux est repris par la ganse rouge de l'uniforme que porte l'ouvreuse: "Dark blue uniform», "red stripe down leg of slacks» (ibid.). Le ledger folio nous apprend encore que le tableau a été achevé le 29 janvier 1939 et que son titre original était non pas New York Movie mais, de façon plus appropriée, New York Movie House (ibid.).

6 D'autres informations précieuses nous sont fournies par les nombreux dessins préparatoires qui ont été conservés et qui illustrent bien la méthode de travail de Hopper. Comme le note Teresa Carbone dans le catalogue de l'exposition, l'architecture de la salle de cinéma, si elle s'inspire principalement du Palace, est en réalité une synthèse, amalgamant des détails croqués par Hopper autour de Noël 1938 dans d'autres salles new-yorkaises, le Strand, le Globe et le Republic (Carbone, 2016, 165-66); on retrouve ici la tendance du peintre à préférer le "type", voire l'archétype, à l'anecdote singulière. Tendance confirmée par le refus formel de Hopper d'identifier la source précise de House by the Railroad ; à John Maass, qui en 1957 demande à Hopper laquelle de deux maisons de Havestraw (New York) il a prise pour modèle, le peintre fait répondre par sa femme: "He did it out of his head. He has seen so many of them » (Levin, 1998, VIII, 17). Il est clair qu'aux yeux de Hopper, ce tableau devenu " iconique " pour avoir à son tour nourri l'inspiration de plusieurs cinéastes américains (Hitchcock, mais aussi George Stevens et Terrence Malick), ${ }^{3}$ constituait une sorte d'hybridation de constructions qu'il avait vues en Nouvelle-Angleterre et en France.

7 Un processus comparable est à l'œuvre dans le traitement de l'ouvreuse, pour laquelle Hopper, selon son habitude, a pris pour modèle sa femme Jo : il a progressivement modifié la position de la figure, qui avait d'abord dans les dessins préparatoires le buste penché, ou encore les jambes légèrement pliées et les bras croisés, ce qui a eu pour effet d'en accentuer le caractère longiligne et la verticalité. ${ }^{4}$ Cette verticalité fait écho à celle du pilier orné, au centre gauche de la composition, avec lequel la fine silhouette de l'ouvreuse cariatide fait par ailleurs le contraste le plus vif. Pour moi ce pilier est l'élément le plus intrigant de la composition. S'il est fort justement décrit dans le ledger folio comme "Gold heavily ordnamented [sic] column L[eft]. centre much muted. [...] Heavy gold column darker, much.» (Lyons, 1997, 50, Foster, 2013, 122), ce pilier suscite triplement la curiosité : par le fait que dans plusieurs dessins préparatoires (Goodrich, 
1970, 130, Foster, 2013, 128, 130, 137, 142), il était dépourvu de toute ornementation ; par sa couleur sombre, qui a pour conséquence qu'on a du mal à démêler ce qu'il représente (une stryge ou un singe accroupi, en bas ?) ; par sa ressemblance peut-être fortuite, mais aussi suggestive que tentante, avec le célèbre trumeau de l'abbatiale romane de Souillac, qui représente la « région de la dissemblance » grouillant d'oiseaux et d'autres animaux monstrueux. ${ }^{5}$

Le trumeau de Souillac (à droite), dessiné par Eugène Viollet-le-Duc $(1866,196)$

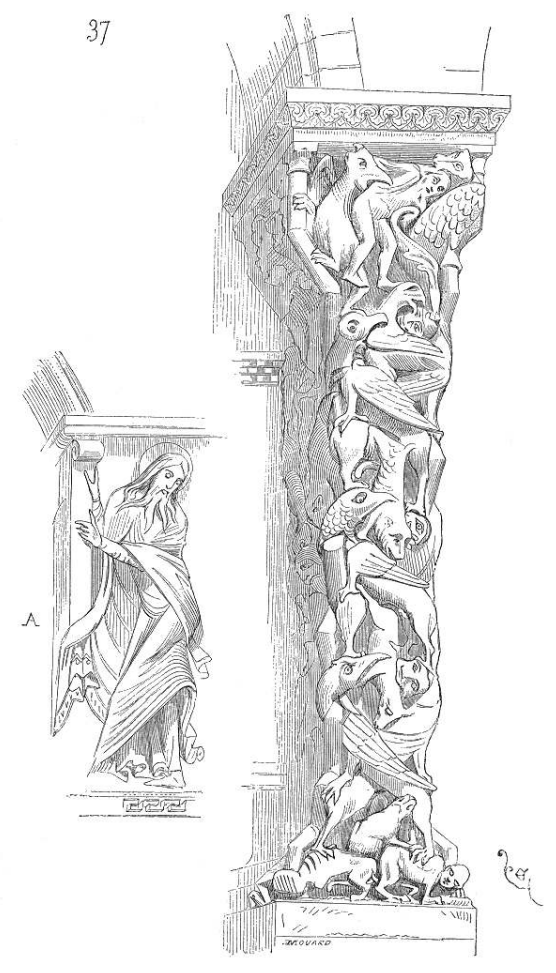

New York Movie invite à une réflexion sur le rapprochement traditionnel, aussi ancien que le cinéma lui-même, entre la situation du spectateur dans la salle de cinéma et celle du rêveur. S'appuyant sur la description de Jo Hopper - "Center grey \& dull white on screen (snowy mountain tips)»-, Teresa Carbone propose d'identifier dans ces sommets enneigés une image tirée du film de Frank Capra, Lost Horizon (Columbia, 1937), adaptation du best-seller de James Hilton et grand film à message pacifiste et utopique dont l'action est située pour l'essentiel au Tibet, dans un décor de hautes montagnes évoquant l'Himalaya (Carbone, 2016, 167). Hypothèse plausible, mieux documentée que celle qu'avait proposée Herbert Ross dans son film Pennies from Heaven (1981), qui constitue un des premiers exemples, avant Les Ailes du désir de Wim Wenders (1987), de «citation» ou de reproduction "à l'identique » d'une toile de Hopper sous forme de tableau vivant dans un film de fiction. Dans Pennies from Heaven, dont l'action est située en 1934, donc au cœur de la Grande Dépression, Ross compose quatre de ces tableaux vivants, inspirés respectivement par deux toiles de Reginald Marsh, Hudson Bay Fur Company et Twenty Cent Movie, et deux toiles de Hopper, New York Movie et Nighthawks. Dans le contexte fictionnel du film, c'est à une projection de Follow the Fleet, comédie musicale de Fred Astaire et Ginger Rogers (réal. Mark Sandrich, RKO, 1936), que sont censés assister les personnages / spectateurs de New York Movie, et ce qu'on voit sur l'écran est un gros plan du couple de danseurs qui s'embrasse. ${ }^{6}$ 
Qu'il s'agisse en effet de sommets neigeux ou d'un tout autre sujet, ce qu'on aperçoit sur le bout d'écran de New York Movie reste en tout cas du domaine de l'informe et de l'impossible à identifier, davantage encore que le pilier qui lui, du moins, est de l'ordre du figuratif, même si cette figuration reste mystérieuse. Impossible donc de savoir si le film fait rêver les deux spectateurs dont on ne voit que le dos. En revanche, nous autres « spectateurs » du tableau de Hopper, « voyeurs » d'une scène qui n'a rien de scabreux, sommes plongés à notre tour dans une rêverie dont l'ouvreuse mélancolique à laquelle nous nous identifions est à la fois l'objet et le sujet. Cette ouvreuse bien nommée (usher) nous conduit jusqu'à un seuil, nous invite à pénétrer dans un monde "autre » qui se distingue de celui du film en ce qu'il est un monde intérieur, onirique. C'est ce qu'André Breton a parfaitement vu et immédiatement ressenti lorsque, peu après son arrivée à New York en juillet 1941, il découvre le tableau au MoMA. Il en parle assez précisément dans un entretien qu'il accorde à la revue surréaliste View d'octobre-novembre, insistant sur l'importance des rideaux grenat, dans lesquels il repère l'indice de la "scène du rêve ", qui est aussi un seuil, un moment décisif de basculement dans un nouveau monde, Éros venant rééquilibrer la part trop généreusement faite à Thanatos par la Seconde Guerre mondiale (Calas, 1941, 1-2).

Plus près de nous, un autre poète, Mark Strand, a su exprimer avec finesse la complexité de notre réaction devant un tableau lui-même complexe, voire contradictoire : "The usherette's preference for privacy over the silver screen, her inwardness, wins our sympathy, despite the fact that our situation as viewers more closely parallels that of the moviegoers. After all, we, too, are looking at a picture. And yet, the way we stand before a painting may resemble more the usherette's stance. Perhaps if I said that we look into instead of at the painting, our sympathy with the inward-looking usherette might be explained. But the truth is we are enacting two contrary impulses - we are looking both at and in, moving between the two as we shift our attention from one side of the canvas to the other " (Strand, 2011, 52).

11 L'épiphanie éprouvée par Breton, l'analyse subtile de Strand, éclairent l'une et l'autre la complexité de l'art de Hopper, sa maîtrise de la composition et de la couleur, sa capacité à engager l'attention du spectateur dans une exploration du tableau et une méditation sur celui-ci qui sont à la fois optiques et cosa mentale, sa capacité enfin à donner au spectateur (un peu à la manière de Vermeer) le sentiment diffus que New York Movie recèle une allégorie, mais que celle-ci demeurera à jamais énigmatique.

\section{BIBLIOGRAPHIE}

CALAS, Nicolas, « Interview with André Breton », View, vol. I, n 7-8, octobre-novembre 1941, 1-2.

CARBONE, Teresa A., « Chair à vif et mauvais genre. Le corps des années 1930 », dans Judith A. Barter, dir., La Peinture américaine des années 1930. The Age of Anxiety, Paris, Musées d'Orsay et de l'Orangerie / Hazan, 2016, 146-71.

FOSTER, Carter E., « New York Movie », dans Carter E. Foster, Hopper Drawing, New York, Whitney Museum of American Art, 2013, 120-49. 
FROMENT, R.P. Jh., Spiritualité d'art roman, Le Plantay (Ain), Abbaye de Notre-Dame des Dombes, 1951.

GOODRICH, Lloyd, Edward Hopper, New York, Abrams, [1970], 1989.

LEVIN, Gail, Hopper's Places, Berkeley, Los Angeles et Londres, University of California Press, $2^{\mathrm{e}}$ éd., 1998.

LYONS, Deborah, Edward Hopper: A Journal of His Work, New York, Whitney Museum of American Art in association with W.W. Norton, 1997.

SCHAPIRO, Meyer, « The Sculptures of Moissac » (1939), dans Meyer Schapiro, Romanesque Art. Selected Papers, New York, George Braziller, 1993, 102-30.

STRAND, Mark, Hopper, New York, Knopf, 2011.

TROYEN, Carol, « Hopper's Women », dans Carol Troyen et al., dir., Edward Hopper, Boston, Museum of Fine Arts, 2007, 177-93.

VIOLLET-LE-DUC, Eugène, Dictionnaire raisonné de l'architecture française du $\mathrm{XI}^{e} a u \mathrm{XVI}^{e}$ siècle, tome VIII, Paris, Bance / Morel, 1866.

\section{NOTES}

1. Liste non exhaustive: New York Movie figure aussi sur la couverture d'Alain Cueff, Edward Hopper, entractes, Paris, Flammarion, 2012, et sur celle de Lawrence Block, dir., In Sunlight or in Shadow. Stories inspired by the paintings of Edward Hopper, New York, Pegasus Books, 2016.

2. Lors d'une visite plus récente au MoMA (juin 2017), la seule toile de Hopper qui était exposée, à nouveau aux côtés de Christina's World, était House by the Railroad (1925), célèbre pour avoir inspiré à Hitchcock le décor de Psycho (1960). Il semble clair qu'aujourd'hui le MoMA, mettant résolument l'accent sur l'art le plus contemporain, a tendance à «abandonner » Hopper au Whitney.

3. Stevens pour la demeure texane de Giant (1956), Malick pour celle de Days of Heaven (1978).

4. Certains de ces dessins préparatoires sont reproduits dans Troyen, 2007, 190, dans Goodrich, 1970, 130, et surtout dans Foster, 2013, 120-49.

5. La « région de la dissemblance » est selon Saint Bernard celle où pénètre l'âme qui franchit le seuil de l'enfer et qui, perdant toute ressemblance divine, devient semblable aux bêtes - voir Froment, 1951, et Schapiro, 1993, 102-30. Je joins le dessin du trumeau de Souillac dans Viollet-leDuc, 1866, 196.

6. En 2002, Todd Haynes fait à son tour revivre New York Movie dans son film Far from Heaven, magnifique pastiche-hommage aux mélodrames des années 1950 de Sirk et Minnelli. En 2013, l'Autrichien Gustav Deutsch réalise l'étonnant Shirley. Visions of Reality, film de fiction structuré par treize tableaux de Hopper, dont New York Movie, où le film projeté sur l'écran devient Dead End de William Wyler (1937). 


\section{AUTEUR}

JEAN-LOUP BOURGET

École normale supérieure (Ulm) 